\title{
On the possibility of measuring the solar oblateness and some relativistic effects from planetary ranging
}

\author{
L. Iorio
}

\author{
Dipartimento di Fisica dell'Università di Bari, via Amendola 173, 70126 Bari, Italy \\ e-mail: lorenzo.iorio@libero.it
}

Received 27 January 2004 / Accepted 3 December 2004

\begin{abstract}
In this paper we first calculate the post-Newtonian gravitoelectric secular rate of the mean anomaly of a test particle freely orbiting a spherically symmetric central mass. Then, we propose a novel approach to suitably combine the presently available planetary ranging data to Mercury, Venus and Mars in order to determine, simultaneously and independently of each other, the Sun's quadrupole mass moment $J_{2 \odot}$ and the secular advances of the perihelion and the mean anomaly. This would also allow to obtain the PPN parameters $\gamma$ and $\beta$ independently. We propose to analyze the time series of three linear combinations of the observational residuals of the rates of the nodes $\dot{\Omega}$, the longitudes of perihelia $\dot{\pi}$ and mean anomalies $\dot{\mathcal{M}}$ of Mercury, Venus and Mars suitably built up in order to absorb the secular precessions induced by the solar oblateness and the post-Newtonian gravitoelectric forces. The values of the three investigated parameters can be obtained by fitting the expected linear trends with straight lines, determining their slopes in arcseconds per century and suitably normalizing them. According to the present-day EPM2000 and DE405 ephemerides accuracy, the obtainable precision would be of the order of $10^{-3}-10^{-4}$ for the PPN parameters and, more interestingly, of $10^{-9}$ for $J_{2 \odot}$. It must be pointed out that the future BepiColombo mission should improve the knowledge of the Mercury's orbit perhaps by one order of magnitude.
\end{abstract}

Key words. relativity - gravitation - celestial mechanics - Sun: fundamental parameters - planets and satellites: general

\section{Introduction}

In this paper we will deal with certain Newtonian and post-Newtonian secular effects affecting the nodes $\Omega$, the longitudes of the perihelia $\pi$ and the mean anomalies $\mathcal{M}$ of the Solar System's planets.

Historically, one of the first classical tests of the Einstein General Theory of Relativity (GTR) was the successful explanation of the anomalous secular perihelion advance of Mercury in the gravitational field of Sun (Einstein 1915). As we will see, this feature of the planetary motion is strictly connected with the problem of the quadrupole mass moment $J_{2 \odot}$ of Sun. In Pireaux \& Rozelot (2003) a theoretical range $J_{2 \odot}=(2 \pm 0.4) \times$ $10^{-7}$ is admitted for the Sun's oblateness. For this topic and the interplay between both effects see the recent review (Pireaux \& Rozelot 2003, and the references therein) and Ciufolini \& Wheeler (1995). Basically, the point is the following. In regard to the secular orbital motions, to which we are interested here, the Sun's mass quadrupole moment induces classical effects which have, qualitatively, the same temporal signature of the relativistic ones. This means that they could corrupt the recovery of the genuine post-Newtonian features of motion because they could not be removed from the time series without removing the post-Newtonian signal of interest as well. All depends on the precision with which $J_{2 \odot}$ is known: should the mismodelling in it induce classical residual precessions larger than the relativistic ones, there would be no hope to get a reliable test of relativistic gravity. As we will see later, the same problems could come from the $N$-body secular precessions. In this paper we propose to disentangle such effects by measuring them in an independent way. More precisely, we propose to extend a certain approach used in Earth artificial satellite motion analysis to the interplanetary arena in order to single out just certain post-Newtonian (and Newtonian) orbital motion features independently of the Parameterized-Post-Newtonian (PPN) framework (Will 1993) which is usually employed in testing competing metric theories of gravity. Instead, the current approach consists of testing the post-Newtonian equations of motion as a whole in terms of the PPN parameters which are determined from multi-parameters fits together with other astrodynamical quantities.

In Table 1 the orbital parameters of the inner planets of the Solar System are reported.

\section{The post-Newtonian effects}

\subsection{The gravitoelectric effects}

In the framework of the standard PPN formalism the post-Newtonian gravitoelectric acceleration induced by the Schwarzschild-like part of the spacetime metric and 
Table 1. Orbital parameters of Mercury, Venus and Mars (http://nssdc.gsfc.nasa.gov/planetary/factsheet/). For the Astronomical Unit (A.U.) we use the value $1 \mathrm{AU}=$ $149597870691 \mathrm{~m}$ of the DE405 (Standish 1998) and EPM2000 ephemerides (Pitjeva 2001a,b). The angle $\epsilon$ refers to the inclination to the ecliptic.

\begin{tabular}{llll}
\hline \hline Planet & $a(\mathrm{AU})$ & $\epsilon\left(^{\circ}\right)$ & $e$ \\
\hline Mercury & 0.38709893 & 7.00487 & 0.20563069 \\
Venus & 0.72333199 & 3.39471 & 0.00677323 \\
Mars & 1.52366231 & 1.85061 & 0.09341233 \\
\hline
\end{tabular}

experienced by a test body freely falling around a static, spherically symmetric central mass $M$ is (Soffel 1989)

$\boldsymbol{a}_{\mathrm{GE}}=\frac{G M}{c^{2} r^{3}}\left\{\left[2(\gamma+\beta) \frac{G M}{r}-\gamma(\boldsymbol{v} \cdot \boldsymbol{v})\right] \boldsymbol{r}+2(1+\gamma)(\boldsymbol{r} \cdot \boldsymbol{v}) \boldsymbol{v}\right\}$,

where $G$ is the Newtonian gravitational constant, $c$ is the speed of light in vacuum, $\boldsymbol{r}$ and $\boldsymbol{v}$ are the position and velocity vectors, respectively, of the test body, $\gamma$ and $\beta$ are the standard Eddington-Robertson-Schiff PPN parameters $(\gamma=\beta=1$ in GTR; in this case Eq. (1) reduces to the expression of Mashhoon et al. 2001). Note that $r$ here is the standard isotropic radial coordinate, not to be confused with the Schwarzschild radial coordinate $r^{\prime}=r\left[1+G M /\left(2 c^{2} r\right)\right]^{2}$. In the usual orbital data reductions it is just $r$ which is employed.

By considering it as a small perturbation to the Newtonian monopole acceleration it is possible to work out its effect on the orbital motion of a test body with the standard perturbative techniques. The secular rate of the argument of pericentre is given by the well known formula

$\dot{\omega}_{\mathrm{GE}}=\frac{3 n G M}{c^{2} a\left(1-e^{2}\right)} v_{\mathrm{GE}}$,

where

$v_{\mathrm{GE}}=\frac{2+2 \gamma-\beta}{3}$,

$a$ and $e$ are the semimajor axis and the eccentricity, respectively, of the test particle's orbit and $n=\sqrt{G M / a^{3}}$ is the (unperturbed) Keplerian mean motion. The orbital period of an unperturbed, two-body Keplerian ellipse is $P=2 \pi / n$. Note that Eq. (2) is an exact result valid to all orders in $e$.

As we will show, also the m ean anomaly $\mathcal{M}$ is affected by a post-Newtonian gravitoelectric secular rate. The Gauss perturbative equation for $\mathcal{M}$ is given by

$\frac{\mathrm{d} \mathcal{M}}{\mathrm{d} t}=n-\frac{2}{n a} A_{R}\left(\frac{r}{a}\right)-\sqrt{1-e^{2}}\left(\frac{\mathrm{d} \omega}{\mathrm{d} t}+\cos i \frac{\mathrm{d} \Omega}{\mathrm{d} t}\right)$,

where $\Omega$ and $i$ are the longitude of the ascending node and the inclination of the test particle's orbit to the equator of the central mass, respectively, and $A_{\mathrm{R}}$ is the radial component of the perturbing acceleration. In order to obtain the post-Newtonian gravitoelectric secular rate of the mean anomaly one must consider, in addition to the pericentre rate of Eq. (2), the radial component of Eq. (1), insert it in the second term of the righthand-side of Eq. (4), evaluate it on the unperturbed Keplerian ellipse, characterized by $r=a\left(1-e^{2}\right) /(1+e \cos f)$ and by the radial and along-track components of the velocity vector which are $v_{R}=n a e \sin f / \sqrt{1-e^{2}}, v_{T}=n a(1+e \cos f) / \sqrt{1-e^{2}}$, respectively ( $f$ is the orbiter's true anomaly), multiplying it by

$\frac{\mathrm{d} t}{P}=\frac{\left(1-e^{2}\right)^{3 / 2} \mathrm{~d} f}{2 \pi(1+e \cos f)^{2}}$,

and integrating over one orbital revolution, i.e. from 0 to $2 \pi$. It turns out that the post-Newtonian gravitoelectric secular rate of the mean anomaly is, to order $O\left(e^{2}\right)$

$\dot{\mathcal{M}}_{\mathrm{GE}} \sim-\frac{n G M}{c^{2} a}\left[(2+4 \gamma+3 \beta)\left(1+\frac{e^{2}}{2}\right)+(2+\gamma) e^{2}\right]$.

We can define

$\mu_{\mathrm{GE}}=\frac{2+4 \gamma+3 \beta}{9}$

It turns out that the second term of Eq. (6) induces for Mercury an additional shift of 1.789 arcseconds/century $\left(" \mathrm{cy}^{-1}\right.$ in the following), while for the other planets it is of the order of $10^{-2}-10^{-4}$ " $\mathrm{cy}^{-1}$. Thus, for them the secular rate of the mean anomaly can be written as

$\dot{\mathcal{M}}_{\mathrm{GE}} \sim-\frac{9 n G M}{c^{2} a \sqrt{1-e^{2}}} \mu_{\mathrm{GE}}$.

However, as we will see later, the mean anomaly of Mercury will not be used in the combined residuals strategy outlined in the following. For Mercury, the post-Newtonian gravitoelectric effect induced by the Sun on $\omega$ and $\mathcal{M}$, according to GTR, amounts to 42.980 " $\mathrm{cy}^{-1}$ and $-127.949{ }^{\prime \prime} \mathrm{cy}^{-1}$, respectively. The most accurate estimate of the gravitoelectric perihelion advance seems to be that obtained for Mercury by E.M. Standish in 2000 with the DE405 ephemerides (Standish 1998) and reported in Pireaux \& Rozelot (2003). He averaged the Mercury's perihelion evolution over two centuries by using the DE405 ephemerides with and without the post-Newtonian accelerations. Standish included in the force models also the solar oblateness with $J_{2 \odot}=2 \times 10^{-7}$, so that the residuals for Mercury accounted for the post-Newtonian effects only; the determined shift was $42.98 \pm 0.0023{ }^{\prime \prime} \mathrm{cy}^{-1}$. The same approach for the mean anomaly (Standish E M private communication) has yielded to $-130.003 \pm 0.0027 "{ }^{\prime \prime} \mathrm{cy}^{-1}$ (See also Table 3 later). Note that the quoted uncertainty do not come from direct observational errors. They depend on the fact that in the force models used in the numerical propagation many astrodynamical parameters occur (masses of planets, asteroids, etc.); their numerical values come from multiparameter fits of real data and, consequently, are affected by observational errors. Such numerical tests say nothing about if GTR is correct or not; they just give an idea of what would be the obtainable accuracy set up by our knowledge of the Solar System arena if the Einstein theory of gravitation would be true.

In regard to the possibility of constructing time series of planetary mean anomalies, it must be noted that a certain effort would be required. Indeed, so far this orbital elements has never been utilized, so that the partials, e.g., should be computed (Pitjeva, private communication 2004). 


\subsection{The gravitomagnetic Lense-Thirring effect}

Another post-Newtonian secular precession which affects not only $\omega$ but also $\Omega$ is the Lense-Thirring effect (Lense \& Thirring 1918) induced by the proper angular momentum $J$ of the central body

$\dot{\omega}_{\mathrm{LT}}=\frac{-6 G J \cos i}{c^{2} a^{3}\left(1-e^{2}\right)^{3 / 2}} \mu_{\mathrm{LT}}$,

$\dot{\Omega}_{\mathrm{LT}}=\frac{2 G J}{c^{2} a^{3}\left(1-e^{2}\right)^{3 / 2}} \mu_{\mathrm{LT}}$,

where

$\mu_{\mathrm{LT}}=\frac{1+\gamma}{2}$.

By assuming for the Sun $J_{\odot}=1.9 \times 10^{48} \mathrm{~g} \mathrm{~cm}^{2} \mathrm{~s}^{-1}$ (Pijpers 2003), the gravitomagnetic effect on, e.g., Mercury's perihelion is of the order of $10^{-3} " \mathrm{cy}^{-1}$ (De Sitter 1916). Such small value is at the edge of the present sensitivity $\left(0.002{ }^{\prime \prime} \mathrm{cy}^{-1}\right)$ in determining the Mercury perihelion shift from the ephemerides (cf. the results obtained by Standish). Moreover, it should be considered that, even if future improvements of the obtainable experimental sensitivity in interplanetary ranging allowed to consider the possibility of measuring the Lense-Thirring perihelion advance of Mercury, the impact of the systematic errors due to the uncertainties in the solar oblateness would severely limit the realistic accuracy obtainable in such demanding measurement. Indeed, by assuming an uncertainty of $\sigma_{J_{2 \odot}}=0.4 \times 10^{-7}$, the error in the secular precession induced by the Sun's quadrupole moment would amount to $245 \%$ of the Lense-Thirring shift of $\pi^{\text {Merc }}$ (see below for the definition of $\pi$ ). Another important source of systematic error in the measurement of such a tiny effect would be represented by the $N$-body classical secular precessions which are of the order of $10^{2}-10^{3} " \mathrm{cy}^{-1}$ (see on the WEB http://ssd.jpl.nasa.gov/elem_planets.html). Indeed, their residual mismodelled part could severely bias the recovery of the Lense-Thirring effect. For a recent approach to these problems and possible strategies to overcome them, see Iorio (2005a). It must be noted that, if, on the other hand, we look at a Lense-Thirring test as a way to measure the Sun's angular momentum by assuming the validity of GTR, a measurement of the solar gravitomagnetic field would have a significance only if the obtainable accuracy was better than $10 \%$; indeed, among other things, the present-day uncertainty in the Sun's angular momentum $J_{\odot}$, which could be measured from the Lense-Thirring precessions, is just of the order of $10 \%$ (Xu \& Ni 1997) in various solar models (Paternó et al. 1996; Elsworth et al. 1995) or even less in the framework of asteroseismogyrometry (Pijpers 2003).

At present, the only performed attempts to explicitly extract the Lense-Thirring signature from the data of orbiting masses in the Solar System are due to Ciufolini and coworkers who analyzed the laser-ranged data of the orbits of the existing LAGEOS and LAGEOS II Earth artificial satellites (Ciufolini et al. 1998). A $20-30 \%$ precision level in measuring the terrestrial gravitomagnetic field is claimed, but other scientists judge these evaluations too optimistic and propose different error budget (Ries et al. 2003). Recent tests
(Ciufolini \& Pavlis 2004), based on an approach proposed in Iorio \& Morea (2004), would have reached the level of accuracy to $10 \%$. However, also in this case there is a controversy about the claimed accuracy (Iorio 2005b). In April 2004 the GP-B spacecraft has been launched. It will carry out a very complex and challenging mission which should be able to measure a gravitomagnetic precession of the spins of four superconducting gyroscopes (Schiff 1960) carried on board at a claimed accuracy of $1 \%$ or better (Everitt et al. 2001).

\section{The solar oblateness}

The solar quadrupole mass moment $J_{2 \odot}$ is an important astrophysical parameter whose precise knowledge could yield many information about the inner structure and dynamics of our star. A reliable evaluation of $J_{2 \odot}$ still faces some controversy: on one side, the theoretical values strongly depend on the solar model used, whereas accurate measurements are very difficult to obtain from observations. For all this matter see the recent review (Pireaux \& Rozelot 2003) and Rozelot et al. (2004). From an observational point of view, $J_{2 \odot}$ is not directly accessible. In this context a dynamical determination of $J_{2 \odot}$, analyzing, e.g., the orbits of the inner planets of the Solar System, is interesting because it might be compared with those derived from solar model dependent values of the oblateness. However, it is not simple to reach this goal because of the interplay between the effects of the solar quadrupole moment with those induced by the post-Newtonian gravitoelectromagnetic forces. Instead of the trajectory of planets, it would be possible to infer $J_{2 \odot}$ from accurate tracking of some drag-free spacecraft orbiting within a few radii of the solar center. This will be the approach followed by, e.g. the BepiColombo mission (see Sect. 4.2). Alternatively, the Sun's quadrupole mass moment can be inferred from in-orbit measurement of solar properties, like the SOHO-MDI space-based observations (Armstrong \& Kuhn 1999), or from Earth-based observations like those realized, e.g., with the scanning heliometer of the Pic du Midi Observatory (Rozelot et al. 2004).

\subsection{The classical precessions induced by the solar oblateness}

For a given planet of the Solar System orbiting the Sun, apart from the classical effects induced by the precession of the equinoxes and by the other planets and major asteroids which are routinely accounted for in the ephemerides computations (Pitjeva 2001a,b), the oblateness of Sun induces also secular precessions on $\Omega, \pi=\omega+\Omega \cos i$ and $\mathcal{M}$ given by

$\dot{\Omega}_{J_{2 \odot}}=-\frac{3}{2} \frac{n J_{2 \odot}}{\left(1-e^{2}\right)^{2}}\left(\frac{R_{\odot}}{a}\right)^{2} \cos i$,
$\dot{\pi}_{J_{2 \odot}}=-\frac{3}{2} \frac{n J_{2 \odot}}{\left(1-e^{2}\right)^{2}}\left(\frac{R_{\odot}}{a}\right)^{2}\left(\frac{3}{2} \sin ^{2} i-1\right)$,
$\dot{\mathcal{M}}_{J_{2 \odot}}=\frac{3}{4} \frac{n J_{2 \odot}}{\left(1-e^{2}\right)^{3 / 2}}\left(\frac{R_{\odot}}{a}\right)^{2}\left(3 \cos ^{2} i-1\right)$. 
Table 2. Post-Newtonian precessions and coefficients of Newtonian precessions of the node, the perihelion and the mean anomaly for Mercury, Venus and Mars in " cy ${ }^{-1}$. The values $\dot{\pi}_{\mathrm{GE}}$, and $\dot{\mathcal{M}}_{\mathrm{GE}}$ are calculated with GTR. The coefficients $\dot{\Omega}_{2 .}, \dot{\pi}_{.2}$ and $\dot{\mathcal{M}}_{2}$ are $\partial\left(\dot{\Omega}_{J_{2 \odot}}\right) / \partial\left(J_{2 \odot}\right)$, $\partial\left(\dot{\pi}_{J_{2 \odot}}\right) / \partial\left(J_{2 \odot}\right)$ and $\partial\left(\dot{\mathcal{M}}_{J_{2}}\right) / \partial\left(J_{2 \odot}\right)$, respectively. In order to have the precessions they must be multiplied by $J_{2 \odot}$. Note that the result for the mean anomaly of Mercury accounts for the correction of order $O\left(e^{2}\right)$ which, instead, can be neglected for the other planets.

\begin{tabular}{lllllll}
\hline \hline Planet & $\dot{\Omega}_{\mathrm{GE}}$ & $\dot{\pi}_{\mathrm{GE}}$ & $\dot{\mathcal{M}}_{\mathrm{GE}}$ & $\dot{\Omega}_{.2}$ & $\dot{\pi}_{.2}$ & $\dot{\mathcal{M}}_{.2}$ \\
\hline Mercury & 0 & 42.981 & -127.949 & -126878.626 & 126404.437 & 123703.132 \\
Venus & 0 & 8.624 & -25.874 & -13068.273 & 13056.803 & 13056.504 \\
Mars & 0 & 1.351 & -4.035 & -980.609 & 980.353 & 976.067 \\
\hline
\end{tabular}

For Mercury ${ }^{1}$ the Newtonian precessions due to Sun oblateness, with $J_{2 \odot}=2 \times 10^{-7}$, are of the order of $10^{-2} " \mathrm{cy}^{-1}$ for $\Omega, \pi$ and $\mathcal{M}$.

\section{The interplay between the solar oblateness and the post-Newtonian precessions}

In Table 2 the relevant parameters of the classical and post-Newtonian secular precessions of the nodes, the perihelia and the mean anomalies of Mercury, Venus and Mars are reported. In all the relativistic tests performed up to now by analyzing the perihelia advances only of the inner planets of the Solar System with the radar ranging technique (Shapiro et al. 1972, 1976; Shapiro 1990) it has been impossible to disentangle the genuine post-Newtonian gravitoelectric contribution of Eq. (2) from the Newtonian precession of Eq. (13). Indeed, the observational residuals of $\dot{\pi}$ for a single planet, built up by suitably switching off the post-Newtonian $O\left(c^{-2}\right)$ terms and the oblateness of Sun in the force models of the equations of motion in the orbital processors softwares, account entirely for the post-Newtonian and the solar oblateness ${ }^{2}$ effects. This is a unsatisfactory situation, both if we are interested in testing post-Newtonian gravity and if we want to obtain a dynamical, model-independent measurement of $J_{2 \odot}$. Indeed, it is, of course, impossible to constraint both the effects if only one perihelion rate is examined one at a time: in recovering one of the two effects we are forced to consider the other one as if it was known. Since the post-Newtonian gravitoelectric effect is three orders of magnitude larger than that induced by solar oblateness, a determination of the latter by assuming the validity of GTR would be affected by a non negligible systematic error induced by the precision to which the post-Newtonian pericentre advance is known from other (more or less indirect and more or less biased by other aliasing effects) tests (Lunar Laser Ranging, binary pulsars periastron advance $\left.{ }^{3}\right)$. The inverse

\footnotetext{
${ }^{1}$ As pointed out in Milani et al. (2002), the angle $i$ refers to the inclination between the planet's orbital plane and the fixed reference plane of the celestial reference frame; it is not the angle $\epsilon$ between the planet's orbital plane and the ecliptic. It turns out that $i \sim \epsilon / 2$. For Mercury $\epsilon=7.00487^{\circ}$.

${ }^{2}$ Also if the solar oblateness is included in the force models, the related uncertainty induces a corresponding systematic error in the recovered post-Newtonian effect. Fortunately, it is small; by assuming $\sigma_{J_{2 \odot}}=0.4 \times 10^{-7}$, it amounts to $0.01 \%$.

${ }^{3}$ Note that the binary pulsars periastron measurement alone should not be considered as a test of relativistic gravity because the masses of the binary system are not known (Stairs et al. 1998); they can be
}

situation is more favorable: indeed, if we are interested in the post-Newtonian gravitoelectric effect the relative systematic error induced on its measurement by the precession due to the solar oblateness amounts to $5 \times 10^{-4}$ even by assuming for the latter effect a $100 \%$ uncertainty $^{4}$.

\subsection{The present-day approach to test post-Newtonian gravity}

At this point it may be interesting to clarify what is the current approach in testing post-Newtonian gravity from planetary data analysis followed by, e.g., the Jet Propulsion Laboratory (JPL). In the interplay between the real data and the equations of motions, which include also the post-Newtonian accelerations expressed in terms of the various PPN parameters, a set of astrodynamical parameters, among which there are also $\gamma$ and $\beta$, are simultaneously and straightforwardly fitted and adjusted and a correlation matrix is also released. This means that the post-Newtonian equations of motion are globally tested as a whole in terms of, among other parameters, $\gamma$ and $\beta$; no attention is paid to this or that particular feature of the post-Newtonian accelerations. The point is that the standard PPN formalism refers to the alternative theories of gravitation which are metric, i.e. based on a symmetric spacetime metric. But it is not proven that an alternative theory of gravitation must necessarily be a metric one. Moreover, even in the framework of the metric alternative theories, the PPN formalism based on 10 parameters is not sufficient to describe every conceivable metric theory of gravitation at the post-Newtonian order; it only describes those theories with a particularly simple post-Newtonian limit. One would, in principle, need an infinite set of new parameters to add to the standard ten parameter PPN formalism in order to describe the post-Newtonian

obtained by assuming the validity of GTR. The Lunar Laser Ranging (LLR) measurements do not allow to single out uniquely the gravitoelctric pericentre advance from the other post-Newtonian features of motion of the Earth-Moon system (Nordtvedt 2001). Indeed, LLR tests the post-Newtonian equations of motion as a whole. Recently, it has been proposed to measure the relativistic gravitoelectric perigee advance of the terrestrial LAGEOS II satellite (Iorio et al. 2002; Lucchesi 2003), but, up to now, the test has not yet been performed.

${ }^{4}$ Note that the impossibility of disentangle the gravitoelectric and Lense-Thirring effects would not seriously affect the recovery of the gravitoelctric precession: indeed, the bias induced by the gravitomagnetic effect on the gravitoelectric shift amounts to $0.004 \%$ only for Mercury. 
approximation of any a priori conceivable metric theory of gravity (Ciufolini 1991; Ciufolini \& Wheeler 1995).

\subsection{The possibilities opened by the future missions}

Concerning the possibility of disentangle the effects of the solar oblateness from those of the post-Newtonian gravitoelectric force, it is stated that the future space mission BepiColombo ${ }^{5}$ of the European Space Agency (ESA) will provide us, among other things, with a dynamical, model-independent and relativity-independent measurement of $J_{2 \odot}$ by measuring with high precision the nodal motion of Mercury (Milani et al. 2002; Pireaux \& Rozelot 2003) which is not affected by the post-Newtonian gravitoelectric force. The claimed accuracy would amount to $\sigma_{J_{2}}=2 \times 10^{-9}$ (Milani et al. 2002). However, such evaluation refers to the formal, statistical obtainable uncertainty only. Indeed, the residuals of the Mercury's node would account, to a certain level of accuracy, for the Lense-Thirring precession as well. By considering such effect as totally unmodelled in the force models, its impact on the measurement of $J_{2 \odot}$ would induce a $8 \times 10^{-9}$ systematic error. The formal, statistical accuracy for $\gamma$ and $\beta$ is evaluated to be of the order of $2 \times 10^{-6}$ (Milani et al. 2002). Also the ESA astrometric mission GAIA ${ }^{6}$ should measure, among other things, the solar quadrupole mass moment by analyzing the longitudes of the ascending nodes of many minor bodies of the Solar System. The obtainable accuracy for $\gamma$ is of the order of $10^{-5}-10^{-7}$ (Vecchiato et al. 2003). The ASTROD mission should be able to measure $J_{2 \odot}$ with a claimed accuracy of the order of $10^{-8}$ or, perhaps, $10^{-9}-10^{-10}$ ( $\mathrm{Ni}$ et al. 2004). The claimed obtainable accuracy for the PPN parameters is $4.6 \times 10^{-7}$ for $\gamma$ and $4 \times 10^{-7}$ for $\beta$. Further improvements may push these limits down to $10^{-8}-10^{-9}$. The recently proposed LATOR mission should be able to measure, among other things, $\gamma$ to a $10^{-8}$ accuracy level and $J_{2 \odot}$ to a $10^{-8}$ level (Turyshev et al. 2004). The solar orbit relativity test SORT (Melliti et al. 2005), which would combine a time-delay experiment with a light deflection test, should allow to reach a $10^{-6}$ accuracy in measuring the PPN parameters.

\section{The experimental accuracy in planetary radar ranging}

Concerning the present-day accuracy of the planetary radar ranging, the radar itself is accurate well below the $100 \mathrm{~m}$ level (Standish 2002). The problem, however, comes from the fact that the surfaces of the planets have large topographical variations. They are modeled in different ways. For Mercury, spherical harmonics and some closure analysis (comparing values when two different measurements reflect off from the same spot on the surface) have been done (Anderson et al. 1996) in DE405. For Venus, a topographical model, which comes from Pettengill et al. (1980), has been used. For Mars,

\footnotetext{
5 See on the WEB http://astro.estec.esa.nl/BepiColombo/ Present ESA plans are for a launch in 2010-2012.

${ }^{6}$ See on the WEB http://astro.estec.esa.nl/GAIA/ Present ESA plans are for a launch in mid-2010.
}

closure points can be used. Closure points are pairs of days during which the observed points on the surface of Mars are nearly identical with respect to their longitudes and latitudes on Mars. Since the same topographical features are observed during each of the two days, the uncertainty introduced by the topography may be eliminated by subtracting the residuals of one day from the corresponding ones of the other day. The remaining difference is then due to only the ephemeris drift between the two days. The closure points for Mars have a priori uncertainties of about $100 \mathrm{~m}$ or less when the points are within 0.2 degrees of each other on the martian surface. Of course, for Mars, there is also the spacecraft ranging - far more accurate than the radar: Viking Landers (1976-82), 10 m; Mars Global Surveyor and Odyssey (1999-2003), 2-3 m. However, correction for Mars topography is possible not only by using closure points (in this method no all observations may be used), but with help of modern hypsometric maps and by the representations of the global topography with an expansion of spherical functions (Pitjeva 2001b). It must now be noted that our knowledge of the orbital motion of Mercury should improve thanks to the future hermean missions Messenger (see on the WEB http://messenger.jhuapl.edu/ and http://discovery.nasa.gov/messenger.html), which has been launched in the summer 2004 and whose encounter with Mercury is scheduled for 2011, and, especially ${ }^{7}$, BepiColombo. A complete error analysis for the range and range-rate measurements can be found in Iess \& Boscagli (2001). According to them, a full 5-way link to the main orbiter will be adopted. A multi-channel combination of the data will allow to remove most of the measurement errors introduced, in a single channel, by the plasma. As a result, a two orders of magnitude improvement in the Earth-Mercury range should be possible, also avoiding the problems related to the surface topography. According to a more conservative evaluation by E.M Standish (Standish, private communication 2004), improvements in the Mercury's orbital parameters might amount to one order of magnitude, i.e. the tens of meters level.

\section{The proposed approach for disentangle the solar oblateness and the post-Newtonian effects}

The strategy proposed here is analogous to that employed for the first time in the LAGEOS-LAGEOS II Lense-Thirring experiment in the gravitational field of Earth by Ciufolini (1996) and, subsequently, extended by Iorio $(2002,2003)$ and Iorio \& Morea (2004).

7 While the spacecraft trajectory will be determined from the range-rate data, the planet's orbit will be retrieved from the range data (Milani et al. 2002). In particular, the determination of the planetary centre of mass is important to this goal which can be better reached by a not too elliptical spacecraft's orbit. The relatively moderate ellipticity of the planned $400 \times 1500 \mathrm{~km}$ polar orbit of BepiColombo, contrary to the much more elliptical path of Messenger, is, then, well adequate. 
Let us assume we have at our disposal $N$ Keplerian orbital elements ${ }^{8} \mathcal{K}$ whose time evolution is affected by a certain number of Newtonian and post-Newtonian effects, say

$\dot{\mathcal{K}}=\dot{\mathcal{K}}_{J_{2}}+\dot{\mathcal{K}}_{\mathrm{GE}}+\dot{\mathcal{K}}_{\mathrm{LT}}+\ldots$

If we are interested in isolating one particular feature, say $\dot{\mathcal{M}}_{\mathrm{GE}}$, and we know it is smaller than other larger effects which affect the same Keplerian element we can explicitly write down the expressions of the observational residuals ${ }^{9} \delta \mathcal{K}_{\text {obs }}$ in term of the feature of interest- which will be assumed to be entirely (or partly) present in the residuals-and of the main larger aliasing effects-which will affect the residuals with their mismodelled part only-so that the number of terms in the sum in the right-hand-side of Eq. (15) which represent the effect of interest and the other most relevant larger bias is equal to the number $N$ of Keplerian orbital elements we have at our disposal. Now we have a system of $N$ equations in $N$ unknowns which we can solve for the effect we are interested in. The resulting expression will be, by construction, independent of the other larger aliasing effects.

Let us apply the previous considerations by writing down the following equations ${ }^{10}$

$$
\begin{aligned}
& \left\{\begin{array}{l}
\delta \dot{\Omega}_{\mathrm{obs}}^{\text {Merc }}=\dot{\Omega}_{.2}^{\text {Merc }} J_{2 \odot}+\dot{\Omega}_{\mathrm{N}-\text { body }}^{\text {Merc }}+\dot{\Omega}_{\mathrm{LT}}^{\text {Merc }} \mu_{\mathrm{LT}}, \\
\delta \dot{\Omega}_{\mathrm{obs}}^{\text {Venus }}=\dot{\Omega}_{.2}^{\text {Venus }} J_{2 \odot}+\dot{\Omega}_{\mathrm{N}-\text { body }}^{\text {Venus }}+\dot{\Omega}_{\mathrm{LT}}^{\text {Venus }} \mu_{\mathrm{LT}}, \\
\delta \dot{\Omega}_{\mathrm{obs}}^{\text {Mars }}=\dot{\Omega}_{.2}^{\text {Mars }} J_{2 \odot}+\dot{\Omega}_{\mathrm{N}-\text { body }}^{\text {Mars }}+\dot{\Omega}_{\mathrm{LT}}^{\text {Mars }} \mu_{\mathrm{LT}},
\end{array}\right. \\
& \left\{\begin{array}{l}
\delta \dot{\mathcal{M}}_{\mathrm{obs}}^{\text {Mars }}=\dot{\mathcal{M}}_{.2}^{\text {Mars }} J_{2 \odot}+\dot{\mathcal{M}}_{\mathrm{GE}}^{\text {Mars }} \mu_{\mathrm{GE}}, \\
\delta \dot{\mathcal{M}}_{\mathrm{obs}}^{\text {Venus }}=\dot{\mathcal{M}}_{.2}^{\text {Venus }} J_{2 \odot}+\dot{\mathcal{M}}_{\mathrm{GE}}^{\text {Venus }} \mu_{\mathrm{GE}},
\end{array}\right. \\
& \left\{\begin{array}{l}
\delta \dot{\pi}_{\mathrm{obs}}^{\mathrm{Mars}}=\dot{\pi}_{.2}^{\mathrm{Mars}} J_{2 \odot}+\dot{\pi}_{\mathrm{GE}}^{\mathrm{Mars}} v_{\mathrm{GE}}, \\
\delta \dot{\pi}_{\mathrm{obs}}^{\mathrm{Merc}}=\dot{\pi}_{.2}^{\mathrm{Merc}} J_{2 \odot}+\dot{\pi}_{\mathrm{GE}}^{\mathrm{Merc}} v_{\mathrm{GE}}
\end{array}\right.
\end{aligned}
$$

where $\delta \dot{\Omega}_{\mathrm{obs}}^{\text {Planet }}, \delta \dot{\pi}_{\mathrm{obs}}^{\text {Planet }}$ and $\delta \dot{\mathcal{M}}_{\mathrm{obs}}^{\text {Planet }}$ are the observational residuals of the rates of the nodes, the longitudes of the perihelia and the mean anomalies of Mercury, Venus and Mars. It is intended

\footnotetext{
8 They could all belong to the same planet or, alternatively, they could all be the same element, say, the node, of $N$ planets or a combination of these possibilities.

9 Here we speak about residuals of Keplerian orbital elements in a, strictly speaking, improper sense. The Keplerian orbital elements are not directly observable: they can only be computed. The basic observable quantities are ranges, range-rates and angles. Here we mean the differences between the time series of $\mathcal{K}$ got from a given observed orbital arc and the time series of $\mathcal{K}$ got from a propagated orbital arc with the force which we are interested in switched off in the force models. The two time series share the same (measured) initial conditions.

${ }^{10}$ Note that in the right-hand-sides of Eqs. (17)-(18) also the mismodelled parts of the classical $N$-body precessions should have been included. Since they are small with respect to the gravitoelectric effects of interest, as we will show later, we can neglect them in the calculations for setting up our combinations. The same also holds for the Lense-Thirring effect in Eq. (18).
}

that all kind of data (optical and radio) would be used. The residuals should be built up by purposely switching off the solar quadrupole moment and the post-Newtonian gravitoelectric accelerations in the force models (or leaving some default values to be subsequently adjusted according to the present strategy) of the orbital processors. Then, the so obtained observational residuals would entirely (or partly, if some default values are left in the force models) adsorb just the investigated secular effects and other post-Newtonian short-periodic features, i.e. not averaged over one orbital revolution of the planet under consideration. In respect to the latter point, it should be noted that the residuals of the mean anomalies would account, e.g., also for the indirect effects on the mean motions $n$ through the perturbations in the semimajor axes $a$

$\Delta n=-\frac{3}{2} \sqrt{\frac{G M}{a^{5}}} \Delta a$.

There are no secular perturbations induced on $a$ by the other planets. If the classical short-periodic effects on $a$ would be of relatively no importance because they would be included in the force models at the best of their accuracy, this is not the case for the post-Newtonian ones. The gravitoelectric field induces no secular variations on $a$, as the classical planetary perturbations. The short-term shift on $a$ can be calculated from Eq. (1) and the Gauss equation for the perturbed rate of semimajor axis

$\frac{\mathrm{d} a}{\mathrm{~d} t}=\frac{2}{n \sqrt{1-e^{2}}}\left[A_{\mathrm{R}} e \sin f+A_{\mathrm{T}}(1+e \cos f)\right]$,

where $A_{\mathrm{T}}$ is the along-track component of the perturbing acceleration. It amounts to

$$
\begin{aligned}
\Delta a_{\mathrm{GE}}= & \frac{G M e}{c^{2}\left(1-e^{2}\right)^{2}}\left[14\left(\cos f_{0}-\cos f\right)\right. \\
& \left.+10 e\left(\cos ^{2} f-\cos ^{2} f_{0}\right)\right]+O\left(e^{3}\right) .
\end{aligned}
$$

For Venus their nominal amplitudes are of the order of $100 \mathrm{~m}$; for Mars they amount to $2 \mathrm{~km}$; Eq. (19) would yield periodic variations whose nominal amplitudes would be of the order of $0.2-1 "{ }^{\prime \prime} \mathrm{cy}^{-1}$ for Venus and Mars, respectively. However, such harmonic signatures could be fitted and removed from the data over sufficiently long time spans. Indeed, since we are interested in the gravitoelectric secular trends on $\pi$ and $\mathcal{M}$ it should be possible, in principle, to construct the residuals by using orbital arcs longer than the sidereal revolution periods of the planets to be used. Then, all the high-frequency perturbations would not affect their time series which should, instead, be characterized by the secular parts of those Newtonian and post-Newtonian features present in the real data but (partly) absent in the force models of the equations of motion in the orbital processors. However, in regard to the possibility of constructing accurate time series of observational residuals many years long the following observations must be kept in mind (Standish 2002). The planetary motions are perturbed by the presence of many asteroids whose masses are quite poorly known. Furthermore, it is not possible to solve for the asteroid masses, other than for the biggest few, because there are too many of them for the data to support such an effort. As a result, 
the ephemerides of the inner planets, especially Mars, will deteriorate over time; the ephemerides have uncertainties at the 1-2 $\mathrm{km}$ level over the span of the observations and growing at the rate of a few $\mathrm{km} / \mathrm{decade}$ outside that span. On the other hand, it must also be noted that the sidereal orbital periods of Venus and Mars amount to 224.701 and 686.980 days, respectively. Another limiting factor in using the mean anomaly is given by the systematic error in the Keplerian mean motion

$\delta n=\frac{\sigma_{G M}}{2 \sqrt{G M a^{3}}}$

due to the uncertainty in the solar $G M$ which is $\sigma_{G M}=$ $8 \times 10^{9} \mathrm{~m}^{3} \mathrm{~s}^{-2}$ (Standish 1995). Its impact on the Keplerian mean motions of Mercury, Venus and Mars amounts to $1.6 \times$ $10^{-2} " \mathrm{cy}^{-1}, 6.3 \times 10^{-3} " \mathrm{cy}^{-1}$ and $2 \times 10^{-3}{ }^{\prime \prime} \mathrm{cy}^{-1}$ respectively.

We can consider Eqs. (16)-(18) as three systems of algebraic linear equations in the three unknowns $J_{2 \odot}, \mu_{\mathrm{GE}}$ and $v_{\mathrm{GE}}$. Their solutions can be written as

$$
\left\{\begin{array}{c}
\delta \dot{\Omega}_{\mathrm{obs}}^{\mathrm{Merc}}+c_{1} \delta \dot{\Omega}_{\mathrm{obs}}^{\mathrm{Venus}}+c_{2} \delta \dot{\Omega}_{\mathrm{obs}}^{\mathrm{Mars}} \\
=J_{2 \odot}\left(\dot{\Omega}_{.2}^{\mathrm{Merc}}+c_{1} \dot{\Omega}_{.2}^{\mathrm{Venus}}+c_{2} \dot{\Omega}_{.2}^{\mathrm{Mars}}\right), \\
\delta \dot{\mathcal{M}}_{\mathrm{obs}}^{\mathrm{Mars}}+c_{1}^{\prime} \delta \dot{\mathcal{M}}_{\mathrm{obs}}^{\mathrm{Venus}} \sim \mu_{\mathrm{GE}}\left(\dot{\mathcal{M}}_{\mathrm{GE}}^{\mathrm{Mars}}+c_{1}^{\prime} \dot{\mathcal{M}}_{\mathrm{GE}}^{\mathrm{Venus}}\right), \\
\delta \dot{\pi}_{\mathrm{obs}}^{\mathrm{Mars}}+c_{1}^{\prime \prime} \delta \dot{\pi}_{\mathrm{obs}}^{\mathrm{Merc}} \sim v_{\mathrm{GE}}\left(\dot{\pi}_{\mathrm{GE}}^{\mathrm{Mars}}+c_{1}^{\prime \prime} \dot{\pi}_{\mathrm{GE}}^{\mathrm{Merc}}\right),
\end{array}\right.
$$

where

$$
\begin{aligned}
& c_{1}=\frac{\dot{\Omega}_{\mathrm{LT}}^{\text {Mars }} \dot{\Omega}_{\mathrm{N}-\text { body }}^{\text {Merc }}-\dot{\Omega}_{\mathrm{LT}}^{\text {Merc }} \dot{\Omega}_{\mathrm{N}-\text { body }}^{\text {Mars }}}{\dot{\Omega}_{\mathrm{LT}}^{\text {Venus }} \dot{\Omega}_{\mathrm{N}-\text { body }}^{\text {Mars }}-\dot{\Omega}_{\mathrm{LT}}^{\text {Mars }} \dot{\Omega}_{\mathrm{N}-\text { body }}^{\text {Venus }}}=-7.73247, \\
& \left\{c_{2}=\frac{\dot{\Omega}_{\mathrm{LT}}^{\text {Merc }} \dot{\Omega}_{\mathrm{N}-\text {-ody }}^{\text {Venus }}-\dot{\Omega}_{\mathrm{LT}}^{\text {Venus }} \dot{\Omega}_{\mathrm{N}-\text { body }}^{\text {Merc }}}{\hat{\Omega}_{\mathrm{LT}}^{\text {Venus }} \Omega_{\mathrm{N}-\text { body }}^{\text {Mars }}-\dot{\Omega}_{\mathrm{LT}}^{\text {Mars }} \Omega_{\mathrm{N}-\text { body }}^{\text {Vens }}}=7.11840\right. \\
& c_{1}^{\prime}=-\dot{\mathcal{M}}_{.2}^{\text {Mars }} / \dot{\mathcal{M}}_{.2}^{\text {Venus }}=-0.07475, \\
& c_{1}^{\prime \prime}=-\dot{\pi}_{.2}^{\mathrm{Mars}} / \dot{\pi}_{.2}^{\mathrm{Merc}}=-0.00775 \text {, }
\end{aligned}
$$

and

$$
\left\{\begin{array}{l}
\dot{\Omega}_{.2}^{\mathrm{Merc}}+c_{1} \dot{\Omega}_{.2}^{\text {Venus }}+c_{2} \dot{\Omega}_{.2}^{\mathrm{Mars}}=-32808.8816^{\prime \prime} \mathrm{cy}^{-1}, \\
\dot{\mathcal{M}}_{\mathrm{GE}}^{\mathrm{Mars}}+c_{1}^{\prime} \dot{\mathcal{M}}_{\mathrm{GE}}^{\mathrm{Venus}}=-2.1007^{\prime \prime} \mathrm{cy}^{-1}, \\
\dot{\pi}_{\mathrm{GE}}^{\mathrm{Mars}}+c_{1}^{\prime \prime} \dot{\pi}_{\mathrm{GE}}^{\mathrm{Merc}}=1.0176^{\prime \prime} \mathrm{cy}^{-1} .
\end{array}\right.
$$

The first equation of Eq. (23) comes from Eq. (16) solved for $J_{2 \odot}$; it allows to obtain $J_{2 \odot}$ independently of the post-Newtonian Lense-Thirring and classical $N$-body secular precessions which would represent the major sources of systematic errors. The second equation of Eq. (23) comes from Eq. (17) solved for $\mu_{\mathrm{GE}}$; it cancels out the secular precessions due to $J_{2 \odot}$ but it is affected by the systematic errors in the Keplerian mean motions $\delta n$. The third equation in Eq. (23) comes from Eq. (18) solved for $v_{\mathrm{GE}}$; it cancels out the secular precessions due to $J_{2 \odot}$. In regard to the impact of the residual mismodelled classical $N$-body precessions on the second and the third combinations of Eq. (23), they should not induce a systematic error larger than the observational one (see below) because the expected values of $\mu_{\mathrm{GE}}$ and $v_{\mathrm{GE}}$ are of the order of unity $^{11}$, contrary to $J_{2 \odot}$ which should be of the order of $10^{-7}$. The adimensional parameters $J_{2 \odot}, \mu_{\mathrm{GE}}$ and $v_{\mathrm{GE}}$ are estimated by fitting the time series of the left-hand-sides of Eq. (23) with straight lines, measuring their slopes, in " $\mathrm{cy}^{-1}$, and, then, by dividing them by the the quantities of Eq. (25) which have the dimensions of " $\mathrm{cy}^{-1}$. Note that the solar quadrupole mass moment would not be affected by the indirect effects on $n$ because only the nodes would be used in its determination. Finally, from the so obtained values of $\mu_{\mathrm{GE}}$ and $v_{\mathrm{GE}}$, which are 1 in GTR and 0 in Newtonian mechanics, it is possible to measure $\gamma$ and $\beta$ independently of the solar oblateness and also of each other as

$$
\left\{\begin{array}{l}
\gamma=\frac{9}{10}\left(\mu_{\mathrm{GE}}+\nu_{\mathrm{GE}}\right)-\frac{4}{5}, \\
\beta=\frac{9}{5} \mu_{\mathrm{GE}}-\frac{6}{5} \nu_{\mathrm{GE}}+\frac{2}{5} .
\end{array}\right.
$$

According to the results of Table 3 it is possible to try to give an estimate of the level of (formal) uncertainty which could be obtained in $J_{2 \odot}, \mu_{\mathrm{GE}}$ and $v_{\mathrm{GE}}$ as

$\left\{\begin{aligned} \sigma_{J_{2 \odot}} & \sim 4.5 \times 10^{-9} \\ \sigma_{\mu_{\mathrm{GE}}} & \sim 7 \times 10^{-4} \\ \sigma_{v_{\mathrm{GE}}} & \sim 3 \times 10^{-5}\end{aligned}\right.$

The evaluation for $\sigma_{\mu_{\mathrm{GE}}}$ is mainly influenced by the systematic errors in the Keplerian mean motions due to $\sigma_{G M}$ according to Eq. (22). It accounts also for the fact that Eq. (19) and the values of $\sigma_{a}$ of Table VI of Pitjeva (2001a) yield a (formal) uncertainty of $3 \times 10^{-5}$. Note that the accuracies in $v_{\mathrm{GE}}$ and $J_{2 \odot}$ would be further improved from the better knowledge of Mercury's orbit which should be obtained from the BepiColombo mission. It should also improve our knowledge of the Sun's GM and, consequently, of $\mu_{\mathrm{GE}}$ as well. The uncertainties in $\gamma$ and $\beta$ would amount to

$\left\{\begin{array}{l}\sigma_{\gamma} \sim 6 \times 10^{-4} \\ \sigma_{\beta} \sim 1 \times 10^{-3}\end{array}\right.$

However, discretion is advised in evaluating the reliability of these results because they refer to the formal, standard statistical errors; realistic errors may be also one order of magnitude larger.

For the most recent determinations of $\gamma$ and $\beta$ we have that, according to the frequency shift of radio photons to and from the Cassini spacecraft, $\sigma_{\gamma}=2.3 \times 10^{-5}$ (Bertotti et al. 2003). This result, combined with $\sigma_{\eta}=4.5 \times 10^{-4}$ for the Strong

\footnotetext{
${ }^{11}$ Let us quantitatively discuss this point. By using the results for the observed centennial rates $\dot{\Omega}$ and $\dot{\varpi}$ released at http://ssd.jpl.nasa.gov/elem_planets.html in, say, the left-hand-side of the third combination of Eq. (23) it is possible to obtain for it a nominal $N$-body shift of $539.6036^{\prime \prime} \mathrm{cy}^{-1}$. The uncertainty in the $N$-body precessions lies mainly in the $\mathrm{Gm}$ of the perturbing planets, among which Jupiter plays the major role. Now, the relative uncertainty in Jupiter's $G m$ is of the order of $10^{-8}$ (Jacobson 2003); then, a reasonable estimate of the order of magnitude of the mismodelled part of the $N$-body shift should be $1 \times 10^{-5} " \mathrm{cy}^{-1}$. This figure must be divided by $1.0176^{\prime \prime} \mathrm{cy}^{-1}$ yielding a relative error in $v_{\mathrm{GE}}$ of the order of $10^{-5}$. Cf. with Eq. (27).
} 
Table 3. Present-day accuracy in determining the node, perihelion and mean anomaly secular rates of Mercury, Venus and Mars according to DE405 (Standish 1998) and EPM2000 (Pitjeva 2001a,b) ephemerides. The figures, in " cy ${ }^{-1}$, represent the formal, statistical errors. Realistic errors should be 10 times larger, at least. While the results by Standish come from the mathematical propagation of the nodes, the perihelia and the mean anomalies (Standish, E. M. private communication) evolution with and without post-Newtonian terms (with $\gamma=\beta=1$ ) and their average over a time span of two centuries, the results by Pitjeva are based on real data. The figure for Mercury has been obtained in 2001, while the other ones have been determined subsequently (Pitjeva, personal communication 2004).

\begin{tabular}{lllll}
\hline \hline Planet & $\sigma_{\dot{\Omega}_{\text {calc }}}($ DE405 $)$ & $\sigma_{\dot{\pi}_{\text {calc }}}($ DE405 $)$ & $\sigma_{\dot{\mathcal{M}}_{\text {calc }}}($ DE405 $)$ & $\sigma_{\dot{\pi}_{\text {obs }}}($ EPM2000 $)$ \\
\hline Mercury & 0.000182 & 0.0023 & 0.0027 & 0.0086 \\
Venus & 0.000006 & 0.0414 & 0.0414 & 0.1037 \\
Mars & 0.000001 & 0.0014 & 0.0014 & 0.0001 \\
\hline
\end{tabular}

Equivalence Principle (SEP) violating parameter $\eta=4 \beta-\gamma-3$ (Nordtvedt 1968a,b) from the most recent analysis of LLR data, yields $\sigma_{\beta}=1 \times 10^{-4}$ (Turyshev et al. 2003). Conservative results from previous LLR analyses yield realistic uncertainties $^{12}$ (Müller et al. 1998) $\sigma_{\gamma}=5 \times 10^{-3}$ and $\sigma_{\beta}=4 \times 10^{-3}$. Recent reanalysis of more than 280000 position observations (1913-2003) of different types including radiometric observations of planets and spacecrafts, CCD astrometric observations of the outer planets and their satellites, meridian transits and photographic observations yield (Pitjeva 2003) $\sigma_{\gamma}=1 \times 10^{-4}$ and $\sigma_{\beta}=1 \times 10^{-4}$. However, as pointed out in (Pitjeva 2003), these uncertainties are formal standard deviations; realistic error bounds may be an order of magnitude larger.

\section{Conclusions}

In this paper we have explicitly worked out the post-Newtonian gravitoelectric secular rate of the mean anomaly of a test particle freely orbiting a spherically symmetric central object. Moreover, we have outlined a possible strategy for determining simultaneously and independently of each other, the solar quadrupole mass moment $J_{2 \odot}$ and two parameters $\nu_{\mathrm{GE}}$ and $\mu_{\mathrm{GE}}$ which account for the post-Newtonian gravitoelectric secular shifts of the perihelion and the mean anomaly, respectively, of planets. They are 0 in Newtonian mechanics and 1 in the General Theory of Relativity. They could be expressed in terms of the standard PPN $\gamma$ and $\beta$ parameters which could, then, be determined independently of each other as well. The usual approach employed in the ephemerides data reductions tests post-Newtonian gravity theories as a whole straightforwardly in terms of the PPN parameters involving the simultaneous fit of many more or less correlated astrodynamical parameters among which there are also $\gamma, \beta$ and $J_{2 \odot}$. In this case, instead, we propose to analyze the time series of three suitably linear combinations of the residuals of $\dot{\Omega}, \dot{\pi}$ and $\dot{\mathcal{M}}$ of Mercury, Venus and Mars built up in order to single out just certain selected Newtonian and post-Newtonian orbital effects. This can be done by setting purposely equal to zero (or to some default values to be subsequently adjusted with the proposed strategy) the orbital effects of interest in the force models of the equations of motion. By suitably choosing the length of the orbital arcs it would be possible to account for the secular terms only. The coefficients of the combinations would make

\footnotetext{
12 The SEP parameter uncertainty in Müller et al. (1998) is $\sigma_{\eta}=9 \times$ $10^{-4}$.
}

each of such combinations sensitive just to one orbital effect at a time, independently of the other ones. By fitting the experimental residual signals with straight lines, measuring their slopes in " $\mathrm{cy}^{-1}$ and suitably normalizing them would yield the values of $J_{2 \odot}, \mu_{\mathrm{GE}}$ and $v_{\mathrm{GE}}$. The obtainable accuracy should be of the order of $10^{-3}-10^{-4}$ for $\gamma$ and $\beta$, accounting also for the systematic errors in the Keplerian mean motions $n$, and, more interestingly, $10^{-9}$ for $J_{2 \odot}$. These estimates should be improved by the future more accurate tracking data of Mercury's orbit from the BepiColombo mission.

Acknowledgements. L. Iorio is grateful to E. M. Standish (JPL) and E. V. Pitjeva (Institute of Applied Astronomy of Russian Academy of Sciences), for their help and useful discussions and clarifications, and to W.-T. Ni (Purple Mountain Observatory) for the updated reference on ASTROD. Thanks also to S.Turyshev (JPL) for his important clarifications on the LATOR mission.

\section{References}

Anderson, J. D., Jurgens, R. F., Lau, E. L., \& Slade, M. A. 1996, Icarus, 124, 690

Armstrong, J., \& Kuhn, J. R. 1999, ApJ, 525, 533

Bertotti, B., Iess, L., \& Tortora, P. 2003, Nature, 425, 374

Ciufolini, I. 1991, Int. J. Mod. Phys. A, 6, 5511

Ciufolini, I., \& Wheeler, J. A. 1995, Gravitation and Inertia (New York: Princeton University Press)

Ciufolini, I. 1996, Il Nuovo Cimento A, 109, 1709

Ciufolini, I., Pavlis, E. C., Chieppa, F., Fernandes-Vieira, E., \& Pérez-Mercader, J. 1998, Science, 279, 2100

Ciufolini, I., \& Pavlis, E. C. 2004, Nature, 431, 958

De Sitter, W. 1916, MNRAS, 76, 699

Einstein, A. 1915, Preuss. Akad. Wiss. Berlin Sitzber., 47, 831

Elsworth, Y., Howe, R., Isaak, G. R., et al. 1995, Nature, 376, 669

Everitt, C. F. W., Buchman, S., DeBra, D. B., \& other members of the Gravity Probe B Team 2001, Gravity Probe B: Countdown to Launch, in Gyros, Clocks, and Interferometers: Testing Relativistic Gravity in Space, ed. C. Lämmerzahl, C. W. F. Everitt, \& F. W. Hehl (Berlin: Springer-Verlag), 52

Iess, L., \& Boscagli, G. 2001, Plan. Space Sci., 49, 1597

Iorio, L. 2002, Class. Quantum Grav., 19, 5473

Iorio, L. 2003, Celest. Mech \& Dyn. Astron., 86, 277

Iorio, L. 2005a, A\&A, 431, 385

Iorio, L. 2005b, New Astron., in press [arXiv: gr-qc/0411024]

Iorio, L., \& Morea, A. 2004, Gen. Rel. Gravit., 36, 1321

Iorio, L., Ciufolini, I., \& Pavlis, E. C. 2002, Class. Quantum Grav., 19, 4301

Jacobson, R. A. 2003, JUP230 orbit solution 
Lense, J., \& Thirring, H. 1918, Phys. Z. 19, 156, translated by Mashhoon, B., Hehl, F. W., \& Theiss, D. S. 1984, Gen. Rel. Grav., 16,711

Lucchesi, D. M. 2003, Phys. Lett. A, 318, 234

Mashhoon, B., Iorio, L., \& Lichtenegger, H. 2001, Phys. Lett. A, 292, 49

Melliti, T., Fridelance, F., \& Samain, E. 2005, in preparation

Milani, A., Vokrouhlický, D., Villani, D., Bonanno, C., \& Rossi, A. 2002, Phys. Rev. D, 66, 082001

Müller, J., Nordtvedt, K., Schneider, M., \& Vokrouhlický, D. 1998, in Proc. of the 11th International Laser Ranging Workshop, Deggendorf, September 21-25, 1998, http://www . wettzell.ifag.de/veranstaltungen/slr/ 11 thlaserworkshop/

Ni, W.-T., Shiomi, S., \& Liao, A.-C. 2004, Class. Quantum Grav., 21, S641

Nordtvedt, K. 1968a, Phys. Rev., 169, 1014

Nordtvedt, K. 1968b, Phys. Rev., 169, 1017

Nordtvedt, K. 2001, An Overview of Solar System Gravitational Physics: The Theory-Experiment Interface, in Gyros, Clocks, and Interferometers: Testing Relativistic Gravity in Space, ed. C. Lämmerzahl, C. W. F. Everitt, \& F. W. Hehl (Berlin: Springer-Verlag), 3

Paternó, S., Sofia, S., \& Di Mauro, M. P. 1996, A\&A, 314, 940

Pettengill, G. H., Eliason, E., Ford, P. G., et al. 1980, J. Geophys. Res., $85, \mathrm{~A} 13,8261$

Pijpers, F. P. 2003, A\&A, 402, 683

Pireaux, S., \& Rozelot, J.-P. 2003, Ap\&SS, 284, 1159

Pitjeva, E. V. 2001a, Cel. Mech. \& Dyn. Astron., 80, 249

Pitjeva, E. V. 2001b, A\&A, 371, 760

Pitjeva, E. V. 2003, invited paper presented at Journées 2003 Astrometry, Geodynamics and Solar System Dynamics: from milliarcseconds to microarcseconds, September 22-25, 2003, Saint-Petersburg, Russia

Ries, J. C., Eanes, R. J., \& Tapley, B. D. 2003, Lense-Thirring Precession Determination from Laser Ranging to Artificial Satellites, in Nonlinear Gravitodynamics. The Lense-Thirring Effect, ed. R. J. Ruffini, \& C. Sigismondi (Singapore: World Scientific), 201
Rozelot, J.-P., Pireaux, S., Lefebvre, S., \& Corbard, T. 2004, preprint [arXiv: astro-ph/0403382]

Schiff, L. I. 1960, Am. J. Phys., 28, 340

Shapiro, I., Pettengill, G. H., Ash, M. E., et al. 1972, Phys. Rev. Lett., 28, 1594

Shapiro, I., Counselman, C., \& King, R. 1976, Phys. Rev. Lett., 36, 555

Shapiro, I. 1990, Solar system tests of general relativity: recent results and present plans, in Proc. of the 12th Int. Conf. on General Relativity and Gravitation, ed. N. Ashby, D. Bartlett, \& W. Wyss (Cambridge: Cambridge University Press), 313

Soffel, M. H. 1989, Relativity in Astrometry, Celestial Mechanics and Geodesy (Heidelberg: Springer Verlag)

Standish, E. M. 1995, in Highlights of Astronomy, ed. I. Appenzeller (Kluwer Academic Publisher)

Standish, E. M. 1998, JPL planetary and lunar ephemerides DE405/LE405, Interoffice Memorandum 312.F-98-048, 1

Standish, E. M. 2002, Future JPL Ephemerides, in Proc. of the IERS Workshop on the Implementation of the New IAU Resolutions, IERS Technical Note No. 29, ed. N. Capitaine, D. Gambis, D. D. McCarthy, G. Petit, J. Ray, B. Richter, M. Rothacher, E. M. Standish, \& J. Vondrak (Verlag des Bundesamts für Kartographie und Geodäsie, Frankfurt am Main), 61

Stairs, I. H., Arzoumanian, Z., Camilo, F., et al. 1998, ApJ, 505, 352

Turyshev, S., Williams, J. G., Nordtvedt, K., Shao, M., \& Murphy, T. W. 2003, 35 Years of Testing Relativistic Gravity: Where do we go from here? Invited talk presented at 302.WE-Heraeus-Seminar: Astrophysics, Clocks and Fundamental Constants, Bad Honnef, Germany, June 16-18, 2003, preprint [arXiv: gr-qc/0311039]

Turyshev, S., Shao, M., \& Nordtvedt, K. 2004, Class. Quantum Grav., 21,2773

Vecchiato, A., Lattanzi, M. G., Bucciarelli, B., et al. 2003, A\&A, 399, 337

Will, C. M. 1993, Theory and Experiment in Gravitational Physics, 2nd edition (Cambridge: Cambridge University Press)

Xu, X., \& Ni, W.-T. 1997, Solar angular momentum calculation, in National Science Council report 